\title{
Halide Ligands to Release Strain in Cadmium Chalcogenide Nanoplatelets and Achieve High Brightness
}

\author{
Marion Dufour ${ }^{1}$, Junling $\mathrm{Qu}^{2}$, Charlie $\mathrm{Greboval}^{2}$, Christophe Méthivier ${ }^{3}$, Emmanuel \\ Lhuillier $^{2}$, Sandrine Ithurria ${ }^{1 *}$ \\ ${ }^{1}$ Laboratoire de Physique et d'Etude des Matériaux, ESPCI-Paris, PSL Research University, \\ Sorbonne Université UPMC Univ Paris 06, CNRS, 10 rue Vauquelin 75005 Paris, France. \\ ${ }^{2}$ Sorbonne Université, CNRS, Institut des NanoSciences de Paris, INSP, F-75005 Paris, \\ France \\ ${ }^{3}$ Laboratoire de Réactivité de Surface, UMR CNRS 7197, Sorbonne Université, UPMC \\ Université Pierre et Marie Curie, Univ Paris 6, 4 place Jussieu, F-75005 Paris, France
}

\begin{abstract}
Zinc blende II-VI semiconductor nanoplatelets (NPLs) are defined at the atomic scale along the thickness of the nanoparticle and are initially capped with carboxylates on the top and bottom [001] facets. These ligands are exchanged on CdSe NPLs with halides that act as X-L-type ligands. These CdSe NPLs are costabilized by amines to provide colloidal stability in nonpolar solvents. The hydrogen from the amine can participate in a hydrogen bond with the lone pair electrons of surface halides. After ligand exchange, the optical features are redshifted. Thus, ligand tuning is another way, in addition to confinement, to tune the optical features of NPLs. The improved surface passivation leads to an increase in the fluorescence quantum efficiency of up to $70 \%$ in the case of bromide. However, for chloride and iodide, the surface coverage is incomplete, and thus, the fluorescence quantum efficiency is lower. This ligand exchange is associated with a decrease in stress that leads to unfolding of the NPLs, which is particularly noticeable for iodide-capped NPLs.
\end{abstract}

Keywords: nanoplatelets, ligands, strain, photoluminescence, halides.

*To whom correspondence should be sent: Sandrine.ithurria@espci.fr 
Because of their atomically precise thickness control, cadmium chalcogenide nanoplatelets ${ }^{1,2}$ (NPLs) have rapidly emerged as a promising material for optoelectronic applications such as field effect transistors, ${ }^{3}$ photodetectors ${ }^{4,5}$ and light emitting diodes. ${ }^{6-8}$

The precise thickness control prevents the appearance of inhomogeneous broadening ${ }^{9}$ and leads to the narrowest photoluminescence linewidth observed among nanocrystals (NCs) $(\approx 9 \mathrm{~nm}$ for an emission at $510 \mathrm{~nm}$ ). In this sense, these materials are even superior to $\mathrm{CsPbBr}_{3}$ perovskite $\mathrm{NCs} .{ }^{10,11}$ They also present a higher crystal stability, with preservation of their crystal structure after washing and ligand exchange. This exceptionally narrow green photoluminescence linewidth makes them especially promising for designing large gamut displays, as shown in Figure 1b. Once combined with a blue InGaN diode and red CdSe/ZnS NPLs, ${ }^{12-14}$ the color range achievable using the NPLs largely surpasses that obtained from the same blue diode coupled with green and red quantum dots (in this case, the photoluminescence linewidth considered is $35 \mathrm{~nm}$ ).

While many efforts have been dedicated to understanding the growth mechanism of these CdSe NPLs, ${ }^{15,16}$ several open questions remain regarding NPL integration. In particular, NPLs suffer from a lack of spectral tunability. The band edge energy can be tuned only in a discrete manner while increasing the thickness. Second, the photoluminescence (PL) efficiency of core-only objects on the order of $20 \%$ remains too weak. These two issues can actually be related to the NPL surface chemistry. This link is obvious in the case of the PL quantum yield (QY), since partial surface passivation is well known to lead to a decrease in the luminescence. ${ }^{17-19}$ In regard to spectral tunability, it has recently been observed in the case of mercury chalcogenide NPLs ${ }^{20,21}$ that one can tune the band edge energy by tuning the capping ligands. These properties directly result from the very strong confinement occurring in NPLs, which induce partial delocalization of the wavefunction on the surface capping molecules. Similar results have been observed on CdSe NPLs capped with thiols. In this case, a clear redshift ${ }^{22}$ is obtained, but it is generally associated with quenching of the PL.

Recently, halide ligands ${ }^{23}$ have attracted significant interest to passivate surface traps of NCs. Another key feature is their shortness, which makes such surface passivation compatible with transport in solar cells ${ }^{24}$ and field effect transistors. ${ }^{25}$ Halide are X-type ${ }^{26}$ ligands, and they have also been grafted onto NPL surfaces to control the PL yield or NPL shape $e^{27,28}$ or to serve as ripening agents to grow thicker NPLs. ${ }^{29,30}$

In this article, we demonstrate the exchange of native carboxylate ligands by halide ligands that are costabilized by a primary amine due to hydrogen bonding on cadmium chalcogenide NPLs. This exchange leads to an increase in the fluorescence efficiency (up to $70 \%$ in toluene) for core-only NPLs. We also demonstrate that halide ligands reduce the stress at the surface of the NPLs, which leads to their unfolding. ${ }^{31,32}$ Finally, the origin of the redshifts of the optical features is discussed, and the NPLs are integrated into light emitting diodes (LEDs). 


\section{RESULTS AND DISCUSSION}

Owen and coworkers ${ }^{26}$ proposed using an organometallic chemistry formalism to discuss the different types of ligands that can bind the surface of NCs. In this covalent bond classification, three types of ligands, denoted $\mathrm{L}, \mathrm{X}$ and $\mathrm{Z}$, are defined depending on the number of electrons provided by the ligating group. A ligand $\mathrm{X}$ (or $\mathrm{L}$ or $\mathrm{Z}$ ) donates 1 electron (or 2 or 0 electrons). As an example, carboxylates and phosphonates are X-type ligands. The zinc blende NPLs present X ligands on their surface (see figure 1a), which ensures their stability and charge compensation.

a.

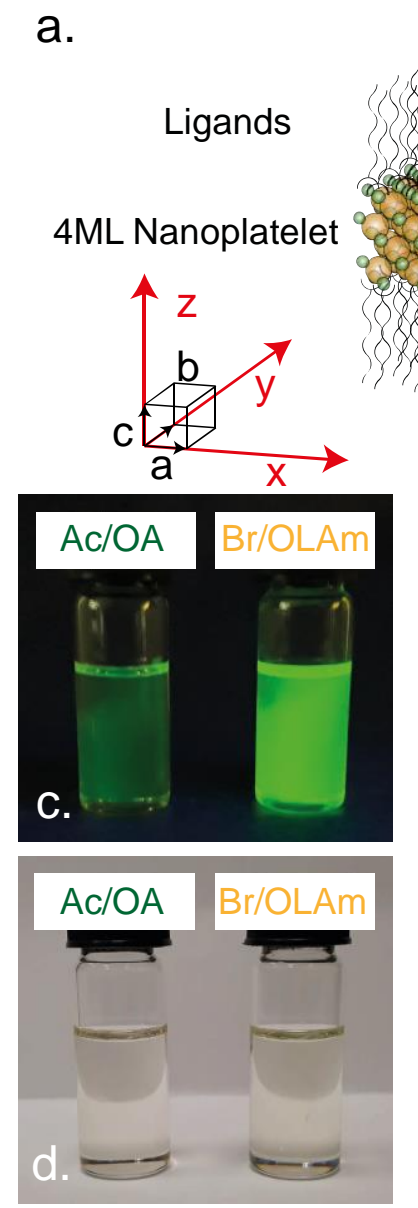

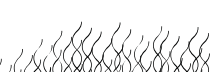

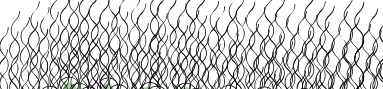
1 1218 2
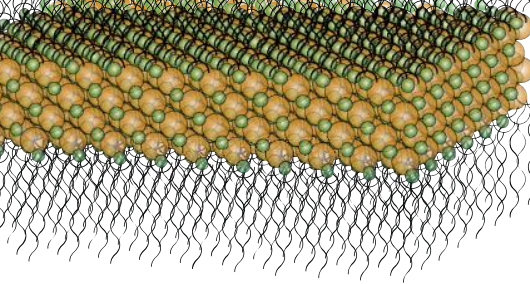

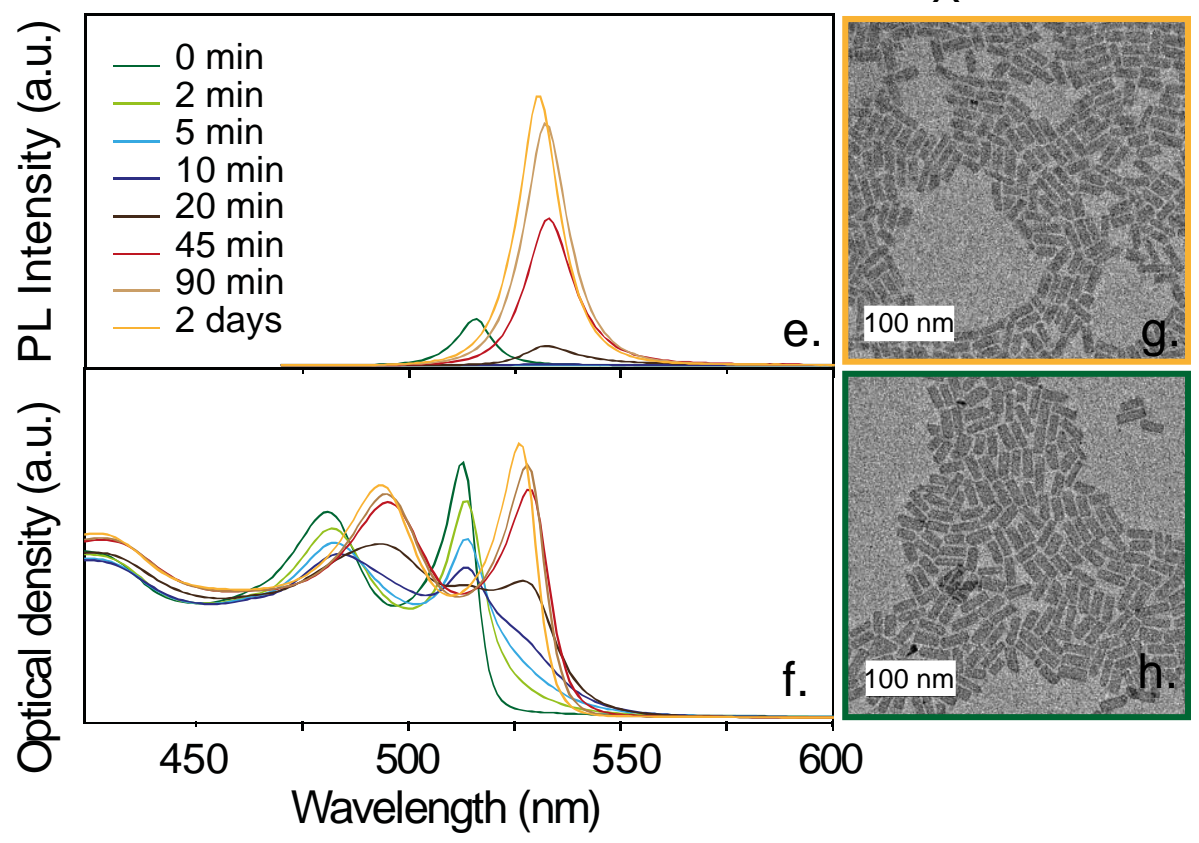

Figure 1: (a.) Scheme of a $4 M L$ CdSe NPL with the top and bottom facet stabilized by X ligands (b.) Chromaticity diagram obtained using a blue InGaN LED combined with CdSe NPLs as the green source and CdSe/ZnS NPLs as the red source. For comparison, the color gamut obtained from spherical QDs has been added. (c.) Picture of a solution of $4 M L C d S e$ NPLs capped by either acetate and oleate (on the left) or bromide and oleylamine (on the right) under UV light in toluene, (d.) Picture of a solution of 4ML CdSe NPLs capped by either acetate and oleate (on the left) or bromide and oleylamine (on the right) (e.) Evolution of the photoluminescence intensity and (f.) the absorption during a ligand exchange from Ac-OA to Br-OLAm. TEM image of 4ML CdSe NPLs capped (g.) by Ac-OA and (h.) by Br-OLAm 
Here, ligand exchanges are performed at room temperature through the introduction of oleylamine (OLAm) and metal halides dissolved in methanol into a solution of NPLs in toluene. In contrast to the optical features of spherical NCs ${ }^{33,34}$ the exchange leads to a significant redshift of the optical features (approximately $80 \mathrm{meV}$ for $4 \mathrm{ML}$ NPLs) (see figure 1e and 1f). The introduction of the ligands $\left(\mathrm{CdBr}_{2}\right.$ and OLAm, denoted hereafter as Br-OLAm) leads to a fast loss of photoluminescence with the appearance of a broad red tail in the absorption spectrum. After 20 minutes, a new absorption transition appears at $\approx 530 \mathrm{~nm}$ with a red tail. This peak blueshifts slightly and sharpens with time. Jointly, the photoluminescence is recovered. After a few hours, the optical features stabilize. Overall, the maximum emission shifts from $514 \mathrm{~nm}$ with acetate and oleate (Ac-OA) to $527 \mathrm{~nm}$ for Br-OLAm ligands. In the case of NPLs, the tuning of ligands, in addition to thickness tunability, is a way to control optical features over the visible range. A slight increase in the full width at half maximum from $9 \mathrm{~nm}$ to $12 \mathrm{~nm}$ (from $42 \mathrm{meV}$ to $53 \mathrm{meV}$ ) was also observed. Such a large shift of the optical features upon changing the surface chemistry from Ac-OA to halide-OLAm has also been observed for CdTe and HgTe NPLs (see Supp Info figure S2 and S5). In the case of 3 ML HgTe NPLs, it was already observed that during the cation exchange, while changing the mercury precursor from $\mathrm{Hg}(\mathrm{Ac})_{2}$ in trioctylamine to $\mathrm{HgCl}_{2}$ in oleylamine, the first excitonic peak was shifted by approximately 100 $\mathrm{nm} .{ }^{35}$ However, for metal telluride NPLs, there is no increase in the PL QY.

In the case of CdSe NPLs, surprisingly, this ligand exchange, contrary to those already performed on zinc blende NPLs, ${ }^{22}$ leads to an increase in the PL QY (see figure 1c and 1d). On average, after ligand exchange with Br-OLAm, the PL QY is approximately $70 \pm 10 \%$. Finally, the $2 \mathrm{D}$ shape of the NPLs is preserved, as observed in the TEM images (figure $1 \mathrm{~g}$ and $1 \mathrm{~h}$ ). Such a high increase in quantum efficiency with $\mathrm{CdX}_{2}$ ligands, called $\mathrm{Z}$ ligands, has recently been reported in CdTe NCs by Houtepen and coworkers. ${ }^{34}$ It should be pointed out that, eventhough the PL QY of Br-OLAm capped CdSe NPLs in solution is high, it is still below $5 \%$ for a film of NPLs. This difference may originate from reabsorption effects arising from small Stokes shift in NPLs associated with the stacking of the NPLs often observed on films (see Supp Info figure S6).
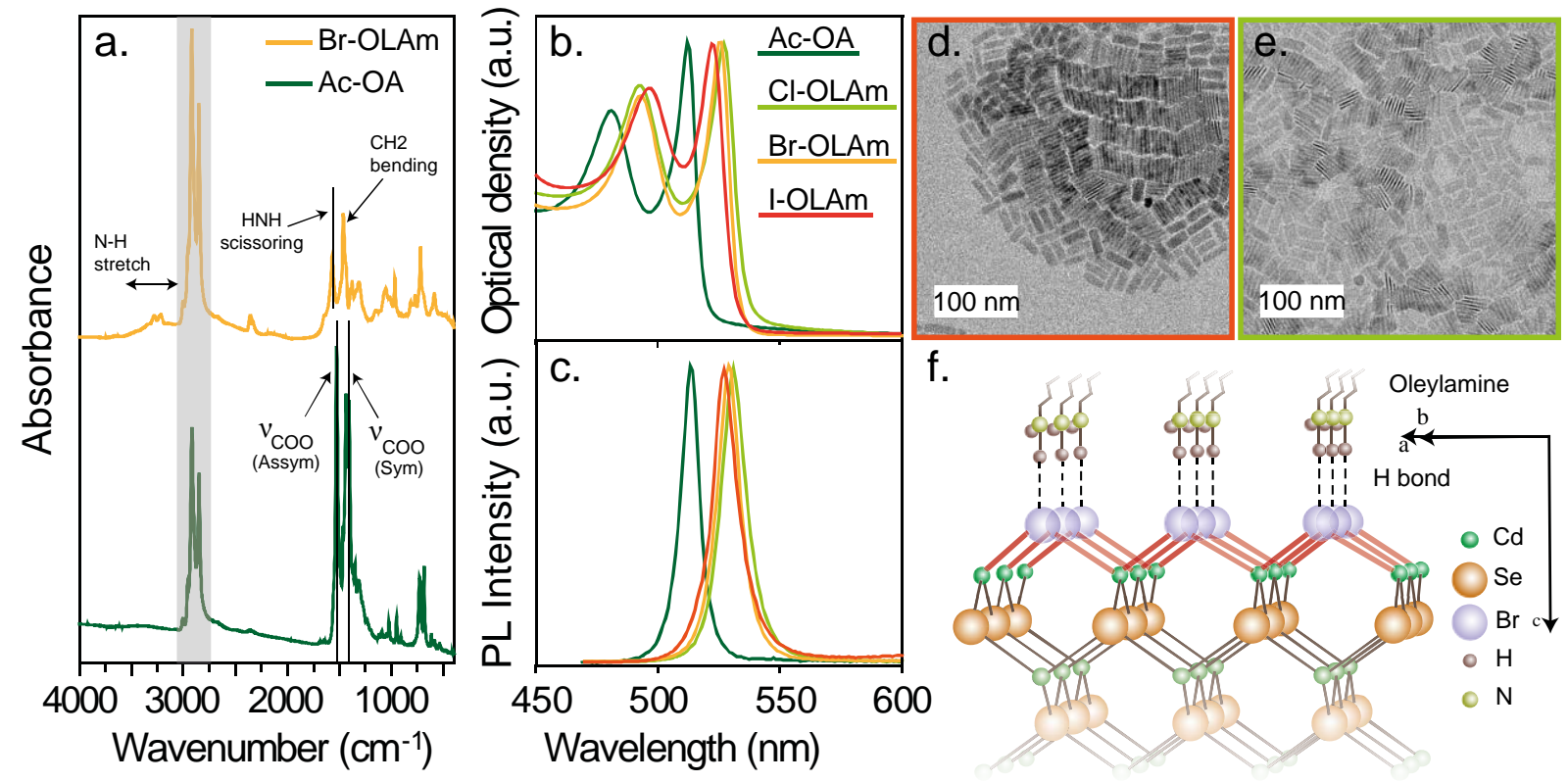

Figure 2: (a.) FTIR spectra of $4 M L C d S e$ NPLs in green for the CdSe/Ac-OA and in orange for the

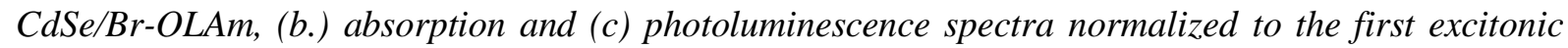
peak and the maximum emission of 4 ML CdSe NPLs capped with Ac-OA, Cl-OLAm, Br-OLAm and IOLAm, (d.) and (e.) TEM images of 4 ML CdSe capped with Cl-OLAm and I-OLAm.(f.) Scheme of the surface of the NPLs with a halide acting as an XL ligand co-stabilized by the amine by hydrogen bonding (dash lines). 
Spherical NCs present different facets on their surface, and hence, it is usually necessary to have a mixture of ligands to efficiently passivate the surface atoms in terms of dangling bonds and charge balance. II-VI semiconductor NPLs are defined at the atomic scale according to their thickness. An NPL of $\mathrm{N}$ monolayers present $\mathrm{N}$ planes of chalcogenide alternating with $\mathrm{N}+1$ planes of cadmium in the [001] direction of the zinc blende structure. Thus, the NPLs present a supplementary plane of cadmium, which is equivalent to having a positive charge on each cadmium from the surface on the top and bottom facets. Each surface cadmium should be passivated by an X-type ligand to ensure the charge neutrality of the NPL.

In 2017, Buhro and coworkers reported ligand exchanges with cadmium halides on wurtzite NPLs. ${ }^{36,37}$ However, under these conditions, instead of introducing X-type ligands, the metal halides act as Ztype ligands. In wurtzite NPLs, the top and bottom facets are neutral with stoichiometric ratios between cadmium and selenium. Thus, the introduction of a Z-type ligand preserves the neutrality of the NPLs while introducing an additional plane of cadmium on the surface. This explains why these authors also observed a larger redshift of the optical features.

Here, the halide ligand acts not only as an X-type ligand but also as an L-type ligand due to its other lone pairs. In the case of a cadmium vacancy on the surface, di-halide cadmium could act as a $\mathrm{Z}$ ligand. The halides can therefore bridge two cadmiums and ensure charge compensation and the tetrahedral environment of the surface cadmiums. For full passivation, the ratio between surface $\mathrm{Cd}$ and halides should be 1:1.

Energy dispersive X-ray spectroscopy (EDX) shows the presence of halides after the exchange. The proportions measured are close to those expected for a halide layer on the top and bottom facets of the NPLs (see table 1). This means that a 4 ML NPL would have 4 planes of Se, 5 planes of Cd and 2 planes of halide after the exchange. However, if we look more closely at the results, the $\mathrm{Cd} / \mathrm{Se}$ ratio is slightly lower than expected for perfectly covered 3 and 4 ML NPLs. However, for all $3 \mathrm{ML}$ and $4 \mathrm{ML}$ CdSe NPLs, the $\mathrm{Cd} / \mathrm{Se}$ ratio is always greater than 1 , meaning the NPLs are still cadmium rich. This result is probably because some $\mathrm{Cd}$ vacancies exist on the surface of halide-capped NPLs. If we consider one halide per surface cadmium considering the vacancies, the bromide-to-cadmium ratio is in close agreement with the expected 1:1 ratio. As an example, in a $10 \mathrm{~nm}$ x $20 \mathrm{~nm} 4 \mathrm{ML}$ CdSe NPL, a $1 \mathrm{~nm}$ thick crown around the NPL already represents more than $1 / 4$ of the surface. Knowing that the ligands close to the edge are weakly bound, ${ }^{18}$ the nonpassivated seleniums are probably on the edges.

For chloride and iodide, the values are also similar, but the halide-to-cadmium ratio is smaller. For iodide, this difference might originate from the larger ionic radius that prevents full coverage of the surface.

Table 1: Molar ratio of 3 and 4 ML CdSe NPLs capped with various ligands: carboxylates, Cl-OLAm, Br-OLAm and I-OLAm.

\begin{tabular}{l|c|c|c} 
& $\mathrm{Cd}(\%)$ & $\mathrm{Se}(\%)$ & $\mathrm{X}^{-}(\%)$ \\
4MLs theory & $\mathbf{5 5 . 6}$ & $\mathbf{4 4 . 4}$ & $<1$ \\
4 ML Ac-OA & $54.4 \pm 1$ & $45.6 \pm 1$ & $\mathbf{1 8 . 2}$ \\
4MLs-X theory & $\mathbf{4 5 . 4}$ & $\mathbf{3 6 . 4}$ & $13.3 \pm 2$ \\
4 ML Cl-OLAm & $46.3 \pm 2$ & $40.6 \pm 2$ & $15.1 \pm 2$ \\
4 ML Br-OLAm & $44.3 \pm 2$ & $40.6 \pm 2$ & $12.6 \pm 2$ \\
4 ML I-OLAm & $45.8 \pm 2$ & $41.6 \pm 2$ & \\
3MLs theory & $\mathbf{5 7 . 2}$ & $\mathbf{4 2 . 8}$ &
\end{tabular}




\begin{tabular}{c|c|c|c} 
3 ML Ac-OA & $57.8 \pm 1$ & $42.2 \pm 1$ & $<1$ \\
3MLs-X theory & $\mathbf{4 4 . 5}$ & $\mathbf{3 3 . 3}$ & $\mathbf{2 2 . 2}$ \\
3 ML Cl-OLAm & $44.4 \pm 2$ & $40.3 \pm 2$ & $15.4 \pm 2$ \\
3 ML Br-OLAm & $40.8 \pm 2$ & $38.9 \pm 2$ & $20.3 \pm 2$ \\
3 ML I-OLAm & $45.4 \pm 1$ & $41.3 \pm 1$ & $13.4 \pm 1$
\end{tabular}

To ensure that the exchange on the surface is predominantly an oleate/ $\mathrm{Br}$ exchange ( $\mathrm{X}$ ligand exchange), the exchanges have been performed not only with $\mathrm{CdX}_{2}$ but also with different metal bromide salts. EDX measurements detect a small amount of the bromide counter cations on the NPLs. The proportions show that less than $10 \%$ of the surface cadmiums have been exchanged by a " $\mathrm{Z}$ ligand exchange" between $\mathrm{Cd}($ oleate) and $\mathrm{MBr}$.

These halide ligands are not sufficient alone to ensure the colloidal stability of NPLs in non-polar solvents. That is why oleylamine is jointly introduced during ligand exchange. Under these conditions, the NPLs are highly stable colloidally, even more stable than as-synthesized NPLs capped with carboxylate, which usually stack with time. Infrared (IR) spectroscopy (see figure 2a) shows the disappearance of the signal attributed to the carboxylate at $1527 \mathrm{~cm}^{-1}$ and $1416 \mathrm{~cm}^{-1}$. It also exhibits peaks at $3180-3310 \mathrm{~cm}^{-1}$, which highlights the presence of the primary amine on the surface of the NPLs. These results were confirmed with photoemission measurements conducted on carboxylates and Br-OLAm-capped 4 ML CdSe NPLs (see Supp Info figure S8 and S9). For carboxylate-capped NPLs, the $\mathrm{C} 1 \mathrm{~s}$ core level provides clear evidence of the presence of aliphatic chain ligands. In addition to the $\mathrm{C} 1 \mathrm{~s}$ contribution at $284.8 \mathrm{eV}$, we observed a second peak at a binding energy (BE) of $288.5 \mathrm{eV}$, which resulted from the carbon of the carboxylic acid group. After ligand exchange, the XPS overview clearly reveals contributions from $\mathrm{Br}$ (see $\mathrm{Br} 3 \mathrm{~d}$ and $3 \mathrm{~s}$ core levels) and from amines (N1s). A careful examination of the N1s state reveals only one contribution at a BE of $399.64 \mathrm{eV}$. This allows us to exclude the presence of ammonium at the interface with bromide, since in this case, a higher $\mathrm{BE}$ in the 401 to $402 \mathrm{eV}$ range would have been expected. ${ }^{38}$ In addition, the $\mathrm{C} 1 \mathrm{~s}$ spectrum does not present a peak at a BE of $288 \mathrm{eV}$, as does the carboxylate capped NPLs. This confirms the removal of the initial carboxylate ligands. The Br-to- $\mathrm{N}$ ratio is estimated to be 1.2 , suggesting a strong complementarity of $\mathrm{Br}$ and oleylamine. The $\mathrm{Br}$-to-Cd ratio is estimated to be 0.3 ( 0.4 would have been expected for full coverage). The Cd:Se ratio determined by XPS (1.3) is quite close to the expected value based on the particle shape (1.25: 5 planes of $\mathrm{Cd}$ for 4 planes of Se) and differs slightly with the one found in EDX. Thus, it still shows that the outer planes of the NPLs are Cd rich and capped with halides.

In the NPLs, the top (001) and bottom (001) facets represent more than $85 \%$ of the surface. Thus, the colloidal stability is driven by the nature of the ligands on these facets. Here, the amine binds to the surface via hydrogen bonds with the lone pair electrons of the surface halide. ${ }^{39}$ The fact that the peaks between 3180 and $3310 \mathrm{~cm}^{-1}$ are broad also suggests the presence of hydrogen bonds. Different primary amines have been tested, and all show the same shift of the optical features (see Supp Info. figure S1). However, the colloidal stability in the nonpolar solvent of the NPLs obtained decreases with the chain length. A schematic overview of the NPL surface includes a layer of halide to ensure the neutrality of the NPLs, costabilized by an amine via hydrogen bonds (see figure 2f.)

The amine also has a second role. Indeed, some experiments have been conducted using butanol to generate hydrogen bonds and stabilize the NPLs in a non-polar solvent (see Supp Info figure S3). These experiments show ligand exchange with a redshift of the absorption. However, the first exitonic peak is redshifted compared to the exchange with OLAm (527 nm for OLAm, $543 \mathrm{~nm}$ with BuOH for 
4 ML NPLs), and the NPLs do not exhibit any photoluminescence. EDX analysis performed on this sample shows an excess of $\mathrm{Cd}$ and $\mathrm{Br}$ compared to $\mathrm{Se}(40.9 \% \mathrm{Cd}, 28.2 \% \mathrm{Br}$ and $30.9 \% \mathrm{Se})$. This suggests that without OLAm, layers of $\mathrm{CdBr}_{2}$ are created instead of only $\mathrm{Br}$ on top of the NPL, which leads to a larger redshift of the absorption and the absence of emission. However, adding OLAm to this solution blueshifts the absorption peak to $527 \mathrm{~nm}$ and significantly increases the QY.

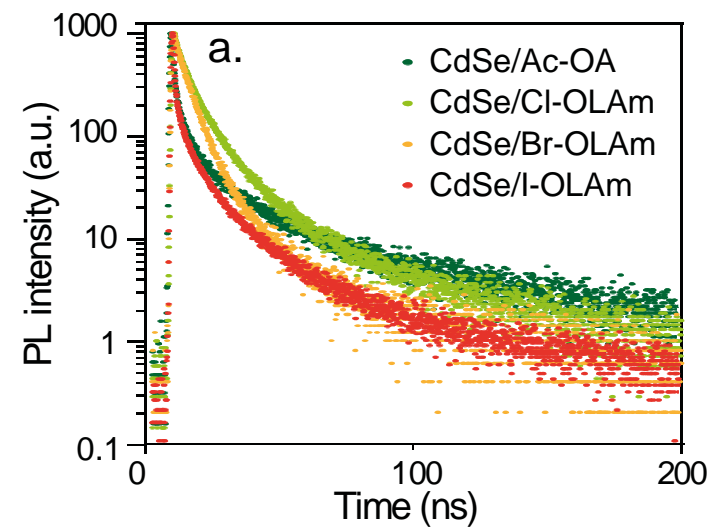

\begin{tabular}{|c|c|c|c|c|c|c|}
\hline & $\tau 1$ & $\%$ & $\tau 2$ & $\%$ & $\tau 3$ & $\%$ \\
\hline $\mathrm{CdSe} / \mathrm{Ac}-\mathrm{OA}$ & 1.0 & 24 & 8.4 & 37 & 55.3 & 39 \\
\hline CdSe/Cl-OLAm & 3.3 & 25 & 10.4 & 59 & 45.6 & 16 \\
\hline CdSe/Br-OLAm & 4.6 & 69 & 12.7 & 25 & 58.4 & 6 \\
\hline CdSe/I-OLAm & 0.7 & 26 & 7.7 & 50 & 41.5 & 24 \\
\hline
\end{tabular}

Figure 3: (a) Photoluminescence decay of 4ML CdSe NPLs capped with Ac-OA and halide-OLAm. (b) Summary of the fitting of the 4 decay rate when fitted with 3 exponentials giving 3 time components (in ns) and their weight in percentage.

PL lifetime measurements were conducted to determine the influence of ligand exchange on the recombination dynamics (figure 3). Two main differences can be seen in figure 3. (i) For Cl-OLAm and Br-OLAm, we observe a loss of the fast $(\sim 1 \mathrm{~ns})$ dynamic. This fast dynamic is often attributed to fast nonradiative recombination due to surface defects. ${ }^{17-19}$ (ii) For all halide-capped NPLs, we also observe a reduction in the longer dynamic present for as-synthesized NPLs ( $\sim 50 \mathrm{~ns})$, which might be attributed to a trapping/detrapping process. ${ }^{40}$ Both of these observations are in accordance with a better surface passivation of Cl-OLAm- and Br-OLAm-capped NPLs and explain the QY increase. However, for I-OLAm, nonradiative processes still occur and are consistent with the low QY observed, which is even lower than that of pristine NPLs. This is characteristic of an incomplete or poor surface passivation and is consistent with the lack of iodide on the surface measured by EDX. When a mixture of Cl-OLAm and I-OLAm is used, it is possible to achieve better passivation and hence a better PL QY than when only I or $\mathrm{Cl}$ is used (see Supp Info figure S4). 
a.

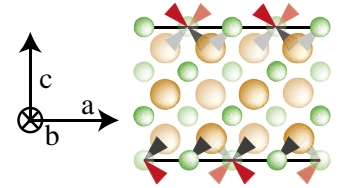

b. TOP facet (001)

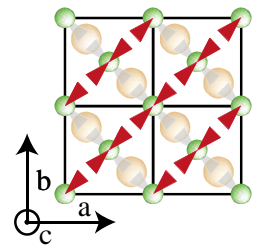

c.

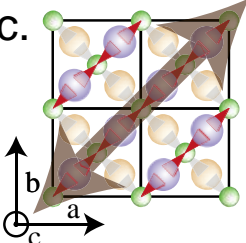

d.

BOTTOM facet $(00 \overline{1})$ for $2 n+1$ MLs NPLs
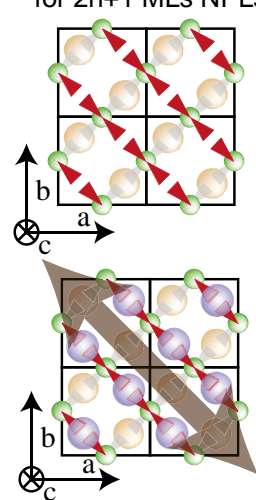

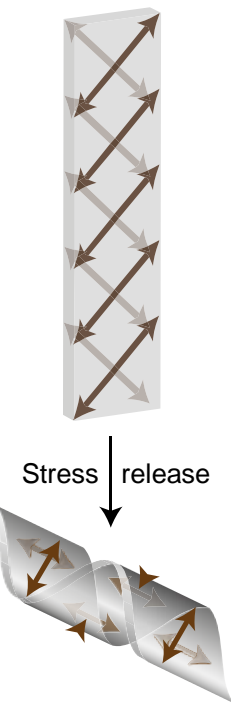

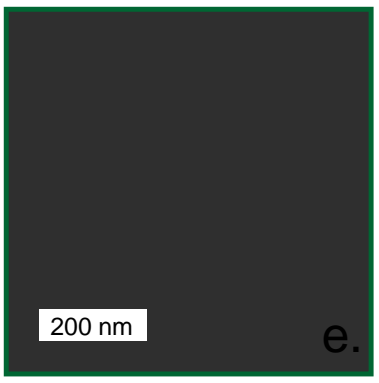
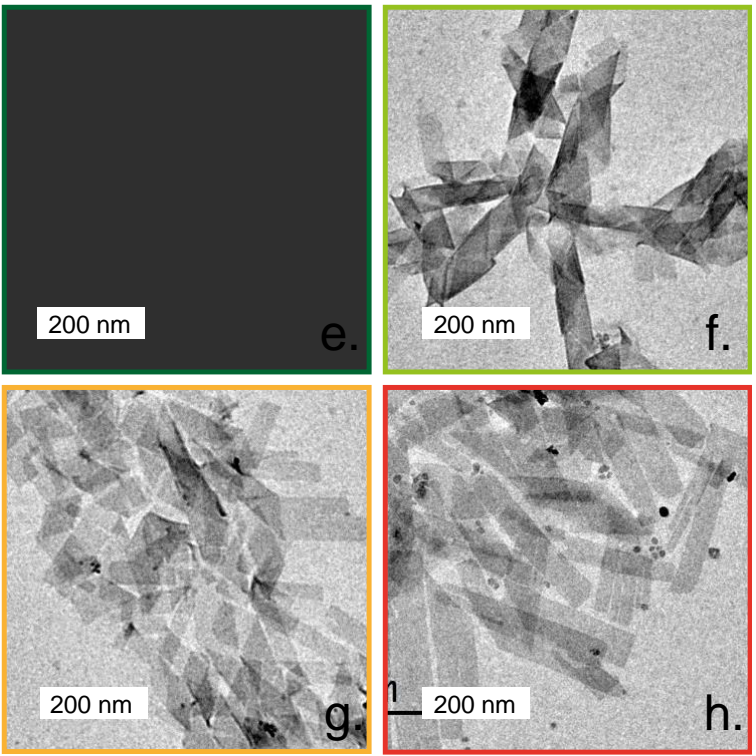

Figure 4: (a.) Side view of the 3ML CdSe NPLs (010) plane. The orange atoms are selenium, and the green ones represent cadmium. The red triangles represent the direction of the bound for an ideal tetrahedral environment (the cadmium dangling bound). (b.) Top and bottom facets of a $3 M L C d S e$ $N P L$. The cadmium environments are equivalent on both facets but rotated at $90^{\circ}$ from each other. (c.) Top and bottom facet of a $3 M L$ CdSe NPL. The blue atoms represent the position of the ligands, which can be halide ligands. The brown arrows indicate the direction of the stress (and hence the strain) on the top and bottom facets. (d.) Scheme of an NPL with the brown arrows indicating the strain and an NPL that has fold as a helix for stress release. (e.), (f.), (g.) and (h.) TEM images of 3 ML CdSe NPLs in dark green for $\mathrm{CdSe} / \mathrm{Ac}-\mathrm{OA}$, in light green the $\mathrm{CdSe} / \mathrm{Cl}-\mathrm{OLAm}$, in light orange the CdSe/Br-OLAm and in red the CdSe/I-OLAm, which show the unfolding of the NPLs.

Jointly with the redshift of the optical features, we observe a change in the relaxed shape of the NPLs (see TEM images in figure 4e-h). First, pristine 3 ML CdSe NPLs are folded as helices. This is a result of strain at the surface of the NPLs. This strain is the result of a tensile stress induced by the ligands on the top and bottom facets of the NPLs. To minimize their energy, the NPLs twist or fold depending on their lateral dimensions. Indeed, in NPLs, the top and bottom facets are chemically equivalent but orientated at $90^{\circ}$ from each other (see figure $3 \mathrm{~b}$ and $3 \mathrm{c}$ ). ${ }^{41}$ This difference comes from the zinc blende structure and the presence of a $\overline{4}$ symmetry axis in the thickness direction. Thus, regarding the rectangular shape of the NPLs and their orientation with thickness, length and width in the <001> direction, the NPLs are chiral NCs. The surface cadmium atoms are linked to two selenium atoms and are missing two atoms to be in their ideal tetrahedral environment. The ligands partially play a role in addition to compensation for the charges. The difference in nature between the core selenium atoms and the atoms from the ligands induces a surface strain whose direction is perpendicular to the direction induced by two selenium atoms in the lower plane. The stress on the top facet is thus in the [110] direction. There is an equivalent stress at the opposite facet but rotated by $90^{\circ}$, which means that on the bottom facet, this stress is in the [1110] direction. To release its internal elastic energy, which contains two contributions: a stretching term and a bending term, ${ }^{42}$ the NPL fold leading to strain that are visible on XRD, with a shift of the peaks compared to the bulk values. In the case of wide NPLs, as for the 3 ML NPLs, the elastic energy is dominated by the stretching term, and the NPLs form a helix (see figure $4 \mathrm{~d}$ ). 
At the end of the synthesis, the native ligands are carboxylate ligands that induce tensile stress in the NPLs, as shown by X-ray diffraction (see figure 5a. in green). Indeed, the CdSe NPL diffractograms exhibit peaks shifted compared to the bulk. In particular, the (220) peak is the superposition of two peaks, a narrow one due to the lattice planes containing the $\mathrm{z}$ axis and thus related to the lateral dimensions and a broad one, which contains the $\mathrm{x}$ or $\mathrm{y}$ axis related to the thickness. ${ }^{22}$ The narrow peak is shifted to smaller angles, showing an average lattice extension of a and b of approximately $6.17 \AA$ instead of $6.05 \AA$ for the zinc blende CdSe structure. Here, we consider a tetragonal distortion, where $\mathbf{a}$ and $\mathbf{b}$ are under tensile stress and $\mathbf{c}$ should be under compressive stress. Such crystal distortion has been reported by Masciocchi and coworkers on PbS nanocrystals, which relax from a rock salt structure to a rhombohedral structure due to ligands. ${ }^{43}$

After ligand exchange with halide-OLAm, the XRD patterns show peaks that are much closer to those expected for bulk zinc blende CdSe with a lattice parameter of $6.05 \AA$ (see figure $5 \mathrm{a}$ and b). In particular, the (200) and (220) thin peaks are related to the planes in the zone with the [001] direction, which is the thickness of the NPLs. Thus, the strain created by the halide ligands is lower than that for Ac-OA ligands. This exchange is accompanied by an unfolding of the NPLs (see figure $4 \mathrm{~h}$ ). Here, the larger the halide is, the stronger the unfolding (size of radii: $\mathrm{r}_{\mathrm{Cl}}<\mathrm{r}_{\mathrm{Br}}<\mathrm{r}_{\mathrm{I}}$ ) (see figure $3 \mathrm{f}$ to $3 \mathrm{i}$ ). The complete unfolding for I-OLAm ligands is suspected to also originate from a lower ligand surface coverage of the NPLs (see table 1). The surface stress is lower, and thus, the NPLs do not need to fold to relax. The nature of the ligands can also have an effect on the stress.
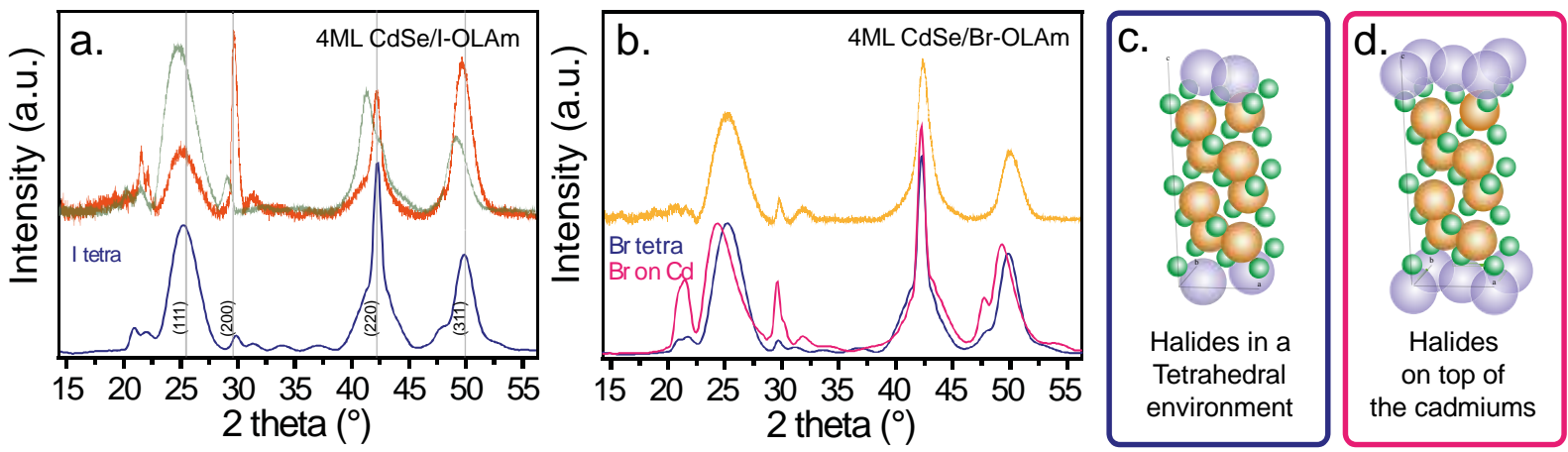

Figure 5:(a.) Experimental X-ray diffractograms of 4ML CdSe NPLs capped with Ac-OA (in green) and with I-OLAm in red. The calculated X-ray pattern of 4MLs CdSe NPLs capped with Iodide bridging between two cadmiums. The gray lines represent the positions of the (111), (200), (220) and (311) peaks of the zinc blende structure with a lattice parameter of $6.05 \AA$ (b.) Experimental X-ray diffractometer of $4 M L$ CdSe NPLs capped with Br-OLAm (in orange). In addition, calculated X-ray patterns of 4 MLs CdSe NPLs capped with bromide bridging between two cadmiums (in blue, same position as the Se) and when bromides are on top of the cadmiums (in pink, distance Cd-Br equal to $2.26 \AA$ ). (c.) and (d.) cells of the 4 ML CdSe NPLs with halides bridging two cadmiums or halides on top of the cadmiums (in green the cadmiums, in orange the selenide and in blue the halides).

The XRD patterns also show peaks that are not expected for a zinc blende $\mathrm{NC}$ at $2 \theta$ equal to 21 and $32^{\circ}$. In the case of Cl-OLAm and Br-OLAm ligands, the peaks at $21.8^{\circ}$ are at the same positions, while in the case of I-OLAm-capped NPLs, this peak is shifted to smaller angles (see Supp Info Figure S10f). These peaks are directly related to the presence of the halides on the NPLs, and they are barely observable when the NPLs are capped with carboxylates. Peng and coworkers ${ }^{44}$ have suggested that contrary to other NCs, due to their thin thickness, it is preferable to define a new cell, where in the $\mathrm{x}$ and $\mathrm{y}$ direction, the lattice parameters are those of the zinc blende structures, but in the $\mathrm{z}$ direction, the lattice parameter is defined by the thickness of the NPL. 
To understand the positions of the halides and their effects on the XRD patterns, we calculated XRD diffractograms by using the Debye scattering equation with atom positions corresponding to a $9 \times 18$ $\mathrm{nm} 4 \mathrm{ML}$ CdSe NPL with a lattice parameter of $6.05 \AA$ capped with halides. The position of the atoms was obtained using Carine crystallography software, and the atomic scattering factor was calculated using values from literature ${ }^{45}$ (see Supp Info for more details). Two cases have been studied: (i) when the halides bridge two cadmiums (as an additional layer of selenium) and (ii) when the halides are positioned vertically on top of the cadmiums (see figure $5 \mathrm{c}$ and $\mathrm{d}$ ).

In case (i), the positions of the peaks are in strong agreement with the I-OLAm-capped NPLs. While the halides are shifted closer to the surface of the NPLs, the first peak between the (200) and (220) peaks is shifted to larger angles but also broadens. When it is moved further, the peaks become narrower but shift to smaller angles (see Supp Info figure S11 to S12). In case (ii), when the Cd-Br distance is equal to $2.27 \AA$, the peak at $21.8^{\circ}$ is close to that observed for Cl-OLAM and Br-OLAm capped NPLs; however, the (111) and (311) peaks are shifted to smaller angles (see Supp Info figure $\mathrm{S} 11$ to S12). If the $\mathrm{Cd}-\mathrm{Br}$ distance is reduced, the peaks indeed shift in the right direction; however, it has no more physical sense since the distance is too small. When this distance is increased, the (111) and (311) peaks shift toward smaller angles compared to the experimental data. Thus, it is likely that bromide and chloride are X-L-type ligands but are not exactly at equal distances between the two cadmiums. The surface cadmiums are probably in a distorted tetrahedral environment but are much less distorted than when they are capped with carboxylate.

By comparing the calculated X-ray and experimental patterns, a difference in the peak intensity is observed (see figure 5a and b) (in the calculation, the Debye factor has not been taken into account, although it introduces a decrease in the peak intensity at wide angles). In particular, the I-OLAmcapped NPLs present a very intense (200) peak in comparison to the (111) peak. The fact that IOLAm-capped NPLs are unfolded also favors their stacking, as can been observed in the TEM image in figure $2 \mathrm{~d}$. Hence, when the NPLs are dropcast on the substrate, they line up either horizontally or vertically when they are stacked. Thus, the NPLs are partially oriented on the substrate, and the planes in the zone with the thickness efficiently diffract the X-rays. This is less noticeable for Cl-OLAm and Br-OLAm NPLs since, as can be observed in the TEM images, they still twist even if the stress is lower than that for carboxylates, as demonstrated by the (220) peaks.

A question that can be raised concerns the origin of the absorption redshift when exchanging acetate/oleate with halides/OLAm. Two complementary effects can be considered: (i) the variation in the thickness due to an extension/contraction of the lattice due to stress and (ii) delocalization of the wavefunction over the ligands. One key point concerning the NPLs is that the energy of the first transition is not proportional to $1 / \mathrm{P}^{2}$ (where $\mathrm{P}$ is the number of planes in the NPL) as expected for an infinite quantum well (see figure 6). Instead, the energy of the first excitonic transition is proportional to 1/P. This observation arises from the large exciton binding energy in the NPLs, the large dielectric confinement, the mirror charges and the quantum confinement and can be anticipated using a tight binding calculation. ${ }^{46,47}$ Here, we have performed ligand exchanges from Ac-OA to Br-OLAm on CdSe NPLs from 2 to $7 \mathrm{MLs},{ }^{46}$ which means from 5 to 15 planes (see Supp Info figure S14). We observe that in both cases, the variation in the first excitonic peak energy is linear with the inverse of the number of planes; however, the slopes are different.

As mentioned previously, the variation in the thickness can explain the first exciton energy shift. When 4 ML CdSe NPLs are capped with carboxylates, the a and $b$ lattice parameters (in the lateral dimensions) are both on average equal to $6.17 \AA$ instead of $6.05 \AA$, which means that using the classical mechanical equation and a Poisson ratio of 0.33 for CdSe, this expansion should lead to a 
contraction of the c parameter to approximately $5.93 \AA$ (see Supp Info). Under these conditions, a 3 ML CdSe NPL would be $13.3 \AA$ A thick instead of $13.6 \AA$ (equivalent to 8.84 planes with a plane of 1.52 $\AA$; see the shift from the dark blue to the blue in figure $6 \mathrm{~b}$ ). For all the NPLs, the XRD patterns enable the determination of the lattice parameter in the lateral dimensions of the NPLs capped with carboxylates and the deduction of their thickness after contraction and the equivalence in terms of the plane's number. In addition to this thickness variation, the strain induces a variation in the material's gap, which can be estimated using Hooke's law $^{48}$ (see Supp Info). The blue squares are shifted down from the green squares. Despite this stress being taken into account, the points are not shifted enough to be on the same slope as those of the halide-capped NPLs. Now, if we consider that CdSe/Br-OLAm are relaxed and that each plane thickness is $1.52 \AA$, it is necessary to add another 0.4 plane ( 2 times 0.2 plane on both sides of the NPL) to align the first exciton energy on the same slope. In other words, the wavefunction is partially delocalized on the layer of halide. Of course, this simple model does not take into account the variation in the exciton binding energy or the variation in the dielectric environment while performing ligand exchange.
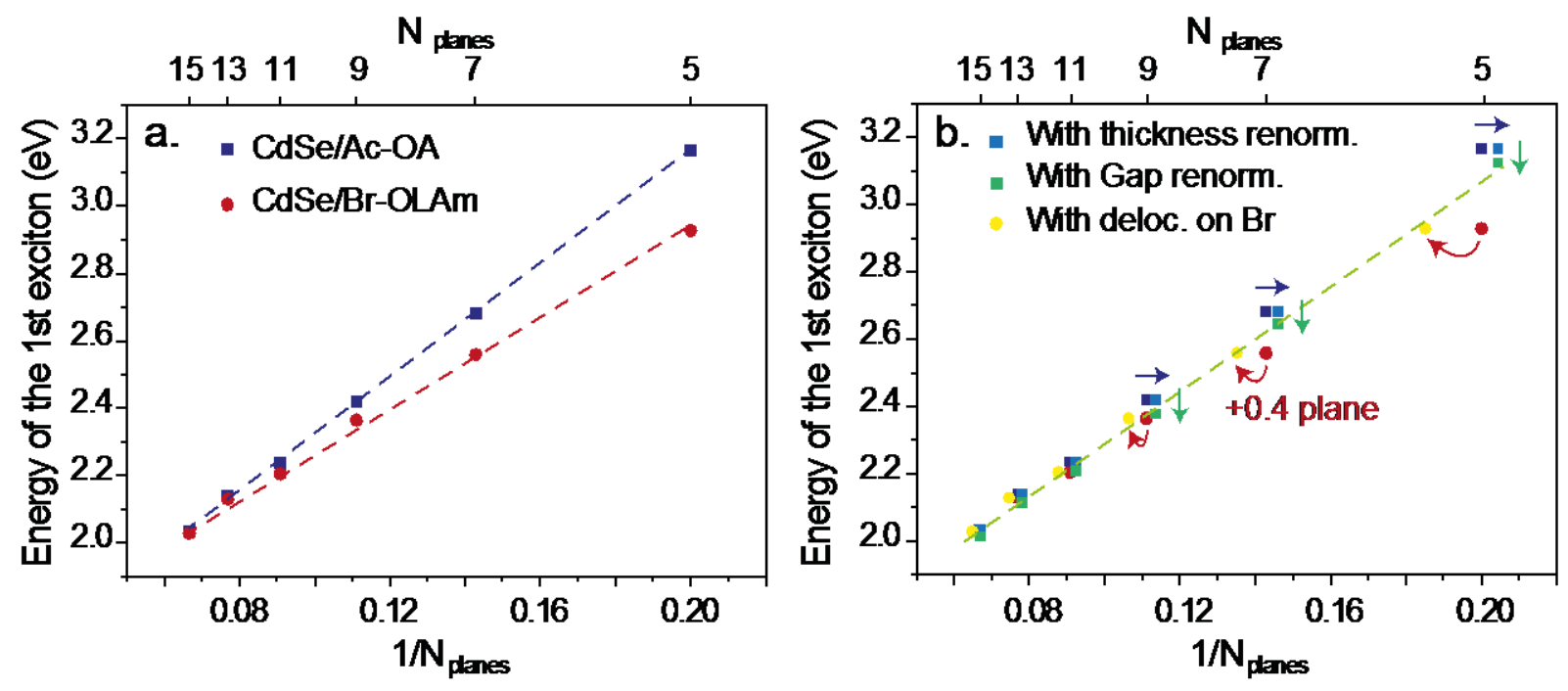

Figure 6: (a.) Variation in the first exciton energy as a function of the inverse of the number of planes in NPLs: dark blue, NPLs capped with carboxylates; dark red, NPLs capped with Br-OLAM. (b.) Variation in the first exciton energy as a function of the inverse of the number of planes in NPLs, while taking into account the compression of the thickness due to tensile stress of the carboxylate ligands (blue) and while taking into account the variation in the gap with the compression (green) or while delocalizing the wavefunction on the equivalent on 0.2 plane on the bromide (in yellow).

Finally, the 4 ML CdSe NPLs have been incorporated into LEDs with a structure proposed by Dai et $a l .{ }^{49}$ The electroluminescence efficiency remains low but was previously optimized for core/shell nanoparticles (see supp info figure S16).

\section{CONCLUSION}

In conclusion, halide ligands can be used as X-L-type ligands for zinc blende II-VI semiconductors. The NPLs are highly stable colloidally in nonpolar solvents due to the costabilization of the surface with amines that bind to the halides via hydrogen bonding. In the case of CdSe NPLs, the Br-OLAm leads to a passivation of traps and thus an increase in the fluorescence QY, which can reach up to 70\%. The halide ligands reduce the stress initially induced by carboxylates and lead to an unfolding of the NPLs, which is complete for iodides. 


\section{ACKNOWLEDEGMENTS}

We thank N. Mezailles for fruitful discussions. This work has been supported by the Region Ile-deFrance in the framework of DIM Nano-K (grant dopQD). This work was supported by French state funds managed by the ANR within the Investissements d'Avenir programme under reference ANR-11IDEX-0004-02, and more specifically within the framework of the Cluster of Excellence MATISSE and also by the grant Nanodose, IPER-Nano2 and H2DH. EL thanks the support ERC starting grant blackQD (grant $n^{\circ}$ 756225). 


\section{METHODS}

\section{CHEMICALS}

Octadecene (ODE) (Aldrich, 90\%), cadmium acetate dihydrate $\left(\mathrm{Cd}(\mathrm{Ac})_{2}\right)$ (Strem, 98\%), cadmium oxide (Strem, 99.99\%), myristic acid (Aldrich, >99\%), oleic acid (OA) (Aldrich 90\%), selenium (Strem Chemicals 99.99\%), tellurium powder (Sigma-Aldrich, 99.99\%), acetone (VWR, 99\%), hexane (VWR, 99\%), ethanol (VWR, 95-97\%), butanol (VWR, $\leq 99.5 \%$ ), cadmium bromide tetrahydrate $\left(\mathrm{CdBr}_{2}\right)$ (Aldrich, $\left.98 \%\right)$, cadmium chloride $\left(\mathrm{CdCl}_{2}\right)$ (Aldrich, beads, $\left.99.999 \%\right)$, cadmium iodide $\left(\mathrm{CdI}_{2}\right)$ (Aldrich, 99\%), propionic acid (Aldrich, 99\%), oleylamine (OLAm) (Acros, 80-90\%), trioctylphosphine (TOP) (Cytec, Solvay), tributylphosphine (TBP) (Cytec, Solvay), potassium bromide (KBr, Sigma Aldrich, 99\%), manganese bromide tetrahydrate $\left(\mathrm{MnBr}_{2}, 4 \mathrm{H}_{2} \mathrm{O}\right.$ Aldrich, $\left.98 \%\right)$, nickel bromide hydrate $\left(\mathrm{NiBr}_{2}, \mathrm{H}_{2} \mathrm{O}\right.$ Aldrich, 98\%), zinc bromide dehydrate $\left(\mathrm{ZnBr}_{2}, 2 \mathrm{H}_{2} \mathrm{O}\right.$ Aldrich, 99\%), ammonium bromide ( $\mathrm{Nh}_{4} \mathrm{Br}$, Sigma Aldrich, 99\%), methanol (VWR, 99.9\%), toluene (VWR, 99.5\%), N-methylformamide (NMF) (Carlo Erba, 99\%), and sodium borohydride $\left(\mathrm{NaBH}_{4}\right)$ (Sigma, $99 \%)$ were used.

\section{PRECURSOR PREPARATION}

Cadmium myristate $\mathrm{Cd}(\mathrm{Myr})_{2}$ : In a $50 \mathrm{~mL}$ three-neck flask, $2.56 \mathrm{~g}$ of $\mathrm{CdO}$ and $11 \mathrm{~g}$ of myristic acid are mixed and degassed for 30 minutes at $80{ }^{\circ} \mathrm{C}$. Then, under argon flow, the temperature is set to 200 ${ }^{\circ} \mathrm{C}$ until the solution becomes colorless (approximately 40 minutes). At $60{ }^{\circ} \mathrm{C}, 30 \mathrm{~mL}$ of methanol is added to solubilize the excess myristic acid. The cadmium myristate is then washed 5 times with methanol. It is finally dried 24 hours under vacuum at $70{ }^{\circ} \mathrm{C}$.

$\mathrm{CdX}_{2} 50 \mathrm{mM}$ solution: $0.05 \mathrm{mmol}$ of $\mathrm{CdX}_{2}(\mathrm{X}=\mathrm{Cl}, \mathrm{Br}, \mathrm{I})$ are added to $1 \mathrm{~mL}$ of $\mathrm{MeOH}$. The solution is sonicated until the full dissolution of the halide precursor.

\section{NANOCRYSTAL SYNTHESIS}

CdSe 3ML synthesis: In a $50 \mathrm{~mL}$ three-neck flask, $240 \mathrm{mg} \mathrm{Cd}(\mathrm{Ac})_{2}$ and $10 \mathrm{~mL}$ ODE are mixed and degassed at $70{ }^{\circ} \mathrm{C}$ for 30 minutes. Then, under Ar flow, the temperature is set to $190{ }^{\circ} \mathrm{C}$. At that temperature, a mixture of $200 \mu \mathrm{L}$ of $\mathrm{OA}, 400 \mu \mathrm{L}$ of TOPSe $1 \mathrm{M}$ and $3.75 \mathrm{~mL}$ of ODE is added dropwise at a rate of $5 \mathrm{~mL} / \mathrm{h}$. Five minutes after the end of the injection, $500 \mu \mathrm{L}$ of OA is added, and the temperature is decreased. The NPLs are precipitated once with $15 \mathrm{~mL}$ of hexane and $15 \mathrm{~mL}$ of ethanol and redispersed in $20 \mathrm{~mL}$ of hexane.

CdSe 4ML synthesis: In a $50 \mathrm{~mL}$ three-neck flask, $24 \mathrm{mg}$ of Se, $340 \mathrm{mg}$ of $\mathrm{Cd}(\mathrm{Myr})_{2}$ and $25 \mathrm{~mL}$ of $\mathrm{ODE}$ are mixed and degassed under vacuum for 15 minutes at room temperature. Under argon flow, the temperature is then set to $240{ }^{\circ} \mathrm{C}$. At $195{ }^{\circ} \mathrm{C}$, when the solution is orange, $110 \mathrm{mg}$ of $\mathrm{Cd}(\mathrm{Acc})_{2}$ are added to the mixture. The temperature is held at $240{ }^{\circ} \mathrm{C}$ for 10 minutes. Then, $500 \mu \mathrm{L}$ of OA is added, and the solution is cooled to room temperature. After adding $25 \mathrm{~mL}$ of hexane and $25 \mathrm{~mL}$ of ethanol, the NPLs are precipitated at $6000 \mathrm{rpm}$ for 5 minutes. Then, the supernatant composed of dots is discarded, and the NPLs are redispersed in $20 \mathrm{~mL}$ of hexane.

Ligand exchange procedure: For 4 ML NPLs, $100 \mu \mathrm{L}$ of a solution of CdSe NPLs is diluted in $3 \mathrm{~mL}$ of toluene. Then, $20 \mu \mathrm{L}$ of OLA is added. Then, $30 \mu \mathrm{L}$ of the halide solution is added. The solution is quickly mixed to homogenize. The amount of halide added is chosen to provide 10 halide anions per surface cadmium. The same procedure has also been performed with higher NPL concentrations. To do so, we increase by a factor of up to 5 all the quantities, except toluene. For 3 ML NPLs, the protocol is the same except that we use $30 \mu \mathrm{L}$ of the NPL solution, $20 \mu \mathrm{L}$ of OLA and $40 \mu \mathrm{L}$ of the 
halide solution (still $10 \mathrm{X} / \mathrm{Cd}_{\text {surf }}$ ). Similarly, we can also exchange in more concentrated solutions. For 2, 5 and $6 \mathrm{ML}$ solutions, we mix $200 \mu \mathrm{L}$ of NPL solution with $50 \mu \mathrm{L}$ of OLA in $3 \mathrm{~mL}$ of toluene and add $100 \mu \mathrm{L}$ of the bromide solution. For EDX, XRD, TEM and FTIR, the NPLs are precipitated once using as little $\mathrm{MeOH}$ as possible and redispersed in toluene.

\section{MATERIAL CHARACTERIZATION}

UV-visible spectra are acquired with a Shimadzu UV-3600 spectrometer. Photoluminescence and excitation spectra are obtained with an Edinburgh Instrument spectrometer. For PL lifetime measurements, a pulsed laser at $375 \mathrm{~nm}$ is used with a repetition time of $500 \mathrm{~ns}$. The photoluminescence quantum yield of NPLs is determined in comparison with Fluorescein reference dye using the following expression:

$\mathrm{QY}_{\mathrm{NPLS}}=\mathrm{QY} \mathrm{P}_{\text {Dye }} \mathrm{X}\left(\mathrm{I}_{\mathrm{NPLS}} / \mathrm{I}_{\text {Dye }}\right) \mathrm{x}\left(\mathrm{OD}_{\text {Dye }} / \mathrm{OD}_{\mathrm{NPLS}}\right) \mathrm{x}\left(\mathrm{n}^{2}{ }_{\mathrm{NPLS}} / \mathrm{n}^{2}{ }_{\text {Dye }}\right)$

where $\mathrm{QY}_{\text {Dye }}$ is the dye quantum yield for a given excitation wavelength, $\mathrm{I}_{\mathrm{QDs}, \mathrm{Dye}}$ is the integrated fluorescence intensity, $\mathrm{OD}_{\mathrm{QDs}, \mathrm{Dye}}$ is the optical density of the QDs and dye solutions and $\mathrm{n}_{\mathrm{QDs}, \text { Dye }}$ is the refractive index of the QDs and dye solutions, respectively.

A JEOL 2010 transmission electron microscope operated at $200 \mathrm{kV}$ is used to observe the nanoparticles. The grids are prepared by dropcasting a solution of NPLs dispersed in toluene in the sub-monolayer regime and degassed overnight under secondary vacuum.

FTIR: A Bruker Vertex 70 in an ATR configuration is used for the measurements. For the measurements, a solution of NPL is dropcast on the diamond cell. The measurement is conducted when the solution is fully dried. Thirty-two spectra are averaged with a $4 \mathrm{~cm}^{-1}$ resolution.

XRD: The sample is prepared by dropcasting a solution of NPLs on a Si wafer. The diffractometer is a Philips X'Pert based on the emission of the $\mathrm{Cu} \mathrm{K}_{\alpha}$ line operated at $40 \mathrm{kV}$ and $40 \mathrm{~mA}$.

EDX: Energy dispersive X-ray (EDX) analysis is conducted by dropcasting a solution of NPLs onto a conductive carbon patch. The measurement is performed using an Oxford EDX probe in an FEI Magellan scanning electron microscope. The current of the beam is $1.6 \mathrm{nA}$, and the operating bias is set to $20 \mathrm{kV}$.

\section{REFERENCES}

(1) Nasilowski, M.; Mahler, B.; Lhuillier, E.; Ithurria, S.; Dubertret, B. Two-Dimensional Colloidal Nanocrystals. Chem. Rev. 2016, 116, 10934-10982.

(2) Lhuillier, E.; Pedetti, S.; Ithurria, S.; Nadal, B.; Heuclin, H.; Dubertret, B. Two-Dimensional Colloidal Metal Chalcogenides Semiconductors: Synthesis, Spectroscopy, and Applications. Acc. Chem. Res. 2015, 48, 22-30.

(3) Lhuillier, E.; Ithurria, S.; Descamps-Mandine, A.; Douillard, T.; Castaing, R.; Xu, X. Z.; Taberna, P. L.; Simon, P.; Aubin, H.; Dubertret, B. Investigating the N- and P-Type Electrolytic Charging of Colloidal Nanoplatelets. J. Phys. Chem. C 2015, 119, 21795-21799.

(4) Lhuillier, E.; Dayen, J. F.; Thomas, D. O.; Robin, A.; Doudin, B.; Dubertret, B. Nanoplatelets Bridging a Nanotrench: A New Architecture for Photodetectors with Increased Sensitivity. Nano Lett. 2015, 15, 1736-1742. 
(5) Lhuillier, E.; Pedetti, S.; Ithurria, S.; Heuclin, H.; Nadal, B.; Robin, A.; Patriarche, G.; Lequeux, N.; Dubertret, B. Electrolyte-Gated Field Effect Transistor to Probe the Surface Defects and Morphology in Films of Thick CdSe Colloidal Nanoplatelets. ACS Nano 2014, 8, 3813-3820.

(6) Giovanella, U.; Pasini, M.; Lorenzon, M.; Galeotti, F.; Lucchi, C.; Meinardi, F.; Luzzati, S.; Dubertret, B.; Brovelli, S. Efficient Solution-Processed Nanoplatelet-Based Light-Emitting Diodes with High Operational Stability in Air. Nano Lett. 2018, 18, 3441-3448.

(7) Fan, F.; Kanjanaboos, P.; Saravanapavanantham, M.; Beauregard, E.; Ingram, G.; Yassitepe, E.; Adachi, M. M.; Voznyy, O.; Johnston, A. K.; Walters, G.; Kim, G.; Lu, Z.; Sargent, E. H. Colloidal CdSe ${ }_{1-\mathrm{X}} \mathrm{S}_{\mathrm{X}}$ Nanoplatelets with Narrow and Continuously-Tunable Electroluminescence. Nano Lett. 2015, 15, 4611-4615.

(8) Chen, Z.; Nadal, B.; Mahler, B.; Aubin, H.; Dubertret, B. Quasi-2D Colloidal Semiconductor Nanoplatelets for Narrow Electroluminescence. Adv. Funct. Mater. 2014, 24, 295-302.

(9) Tessier, M. D.; Javaux, C.; Maksimovic, I.; Loriette, V.; Dubertret, B. Spectroscopy of Single CdSe. ACS Nano 2012, 6, 6751-6758.

(10) Protesescu, L.; Yakunin, S.; Bodnarchuk, M. I.; Krieg, F.; Caputo, R.; Hendon, C. H.; Yang, R. X.; Walsh, A.; Kovalenko, M. V. Nanocrystals of Cesium Lead Halide Perovskites (CsPbX3, $\mathrm{X}=\mathrm{Cl}, \mathrm{Br}$, and I): Novel Optoelectronic Materials Showing Bright Emission with Wide Color Gamut. Nano Lett. 2015, 15, 3692-3696.

(11) Canneson, D.; Shornikova, E. V.; Yakovlev, D. R.; Rogge, T.; Mitioglu, A. a.; Ballottin, M. V.; Christianen, P. C. M.; Lhuillier, E.; Bayer, M.; Biadala, L. Negatively Charged and Dark Excitons in $\mathrm{CsPbBr} 3$ Perovskite Nanocrystals Revealed by High Magnetic Fields. Nano Lett. 2017, 17, 6177-6183.

(12) Cruguel, H.; Livache, C.; Martinez, B.; Pedetti, S.; Pierucci, D.; Izquierdo, E.; Dufour, M.; Ithurria, S.; Aubin, H.; Ouerghi, A.; Lacaze, E.; Silly, M. G.; Dubertret, B.; Lhuillier, E. Electronic Structure of CdSe-ZnS 2D Nanoplatelets. Appl. Phys. Lett. 2017, 110, 1-5.

(13) Polovitsyn, A.; Dang, Z.; Movilla, J. L.; Martín-García, B.; Khan, A. H.; Bertrand, G. H. V.; Brescia, R.; Moreels, I. Synthesis of Air-Stable CdSe/ZnS Core-Shell Nanoplatelets with Tunable Emission Wavelength. Chem. Mater. 2017, 29, 5671-5680.

(14) Feng, F.; Nguyen, L. T.; Nasilowski, M.; Nadal, B.; Dubertret, B.; Maître, A.; Coolen, L. Probing the Fluorescence Dipoles of Single Cubic CdSe/CdS Nanoplatelets with Vertical or Horizontal Orientations. ACS Photonics 2018, 5, 1994-1999.

(15) Riedinger, A.; Ott, F. D.; Mule, A.; Mazzotti, S.; Knüsel, P. N.; Kress, S. J. P.; Prins, F.; Erwin, S. C.; Norris, D. J. An Intrinsic Growth Instability in Isotropic Materials Leads to Quasi-TwoDimensional Nanoplatelets. Nat. Mater. 2017, 16, 743-748.

(16) Chen, Y.; Chen, D.; Li, Z.; Peng, X. Symmetry-Breaking for Formation of Rectangular CdSe Two-Dimensional Nanocrystals in Zinc-Blende Structure. J. Am. Chem. Soc. 2017, 139, 10009-10019.

(17) Yadav, S.; Singh, A.; Thulasidharan, L.; Sapra, S. Surface Decides the Photoluminescence of Colloidal CdSe Nanoplatelets Based Core/Shell Heterostructures. J. Phys. Chem. C 2018, 122, 820-829. 
(18) Singh, S.; Tomar, R.; Ten Brinck, S.; De Roo, J.; Geiregat, P.; Martins, J. C.; Infante, I.; Hens, Z. Colloidal CdSe Nanoplatelets, A Model for Surface Chemistry/Optoelectronic Property Relations in Semiconductor Nanocrystals. J. Am. Chem. Soc. 2018, 140, 13292-13300.

(19) Houtepen, A. J.; Hens, Z.; Owen, J. S.; Infante, I. On the Origin of Surface Traps in Colloidal II-VI Semiconductor Nanocrystals. Chem. Mater. 2017, 29, 752-761.

(20) Izquierdo, E.; Dufour, M.; Chu, A.; Livache, C.; Martinez, B.; Amelot, D.; Patriarche, G.; Lequeux, N.; Lhuillier, E.; Ithurria, S. Coupled HgSe Colloidal Quantum Wells through a Tunable Barrier: A Strategy to Uncouple Optical and Transport Band Gap. Chem. Mater. 2018, $30,4065-4072$

(21) Izquierdo, E.; Robin, A.; Keuleyan, S.; Lequeux, N.; Lhuillier, E.; Ithurria, S. Strongly Confined HgTe 2D Nanoplatelets as Narrow Near-Infrared Emitters. J. Am. Chem. Soc. 2016, $138,10496-10501$.

(22) Antanovich, a.; Achtstein, a. W.; Matsukovich, a.; Prudnikau, a.; Bhaskar, P.; Gurin, V.; Molinari, M.; Artemyev, M. A Strain-Induced Exciton Transition Energy Shift in CdSe Nanoplatelets: The Impact of an Organic Ligand Shell. Nanoscale 2017, 9, 18042-18053.

(23) Ghosh, S.; Manna, L. The Many "Facets" of Halide Ions in the Chemistry of Colloidal Inorganic Nanocrystals. Chem. Rev. 2018, 118, 7804-7864.

(24) Tang, J.; Kemp, K. W.; Hoogland, S.; Jeong, K. S.; Liu, H.; Levina, L.; Furukawa, M.; Wang, X.; Debnath, R.; Cha, D.; Chou, K. W.; Fischer, A.; Amassian, A.; Asbury, J. B.; Sargent, E. H. Colloidal-Quantum-Dot Photovoltaics Using Atomic-Ligand Passivation. Nat. Mater. 2011, 10, 765-771.

(25) Norman, Z. M.; Anderson, N. C.; Owen, J. S. Electrical Transport and Grain Growth in Solution-Cast, Chloride-Terminated Cadmium Selenide Nanocrystal Thin Films. ACS Nano 2014, 8, 7513-7521.

(26) Anderson, N. C.; Hendricks, M. P.; Choi, J. J.; Owen, J. S. Ligand Exchange and the Stoichiometry of Metal Chalcogenide Nanocrystals: Spectroscopic Observation of Facile Metal-Carboxylate Displacement and Binding. J. Am. Chem. Soc. 2013, 135, 18536-18548.

(27) Gerdes, F.; Klein, E.; Kull, S.; Ramin Moayed, M. M.; Lesyuk, R.; Klinke, C. Halogens in the Synthesis of Colloidal Semiconductor Nanocrystals. Zeitschrift für Phys. Chemie 2018, 232, $1267-1280$.

(28) Gerdes, F.; Navío, C.; Juárez, B. H.; Klinke, C. Size, Shape, and Phase Control in Ultrathin CdSe Nanosheets. Nano Lett. 2017, 17, 4165-4171.

(29) Christodoulou, S.; Climente, J. I.; Planelles, J.; Brescia, R.; Prato, M.; Martin-Garcia, B.; Khan, A. H.; Moreels, I. Chloride-Induced Thickness Control in CdSe Nanoplatelets. Nano Lett. 2018, 18, 6248-6254.

(30) Cho, W.; Kim, S.; Coropceanu, I.; Srivastava, V.; Diroll, B. T.; Hazarika, A.; Fedin, I.; Galli, G.; Schaller, R. D.; Talapin, D. V. Direct Synthesis of Six-Monolayer (1.9 nm) Thick ZincBlende CdSe Nanoplatelets Emitting at 585 nm. Chem. Mater. 2018, 30, 6957-6960. 
(31) Hutter, E. M.; Bladt, E.; Goris, B.; Pietra, F.; Bok, J. C. Van Der; Boneschanscher, M. P.; Donega, C. D. M.; Bals, S.; Vanmaekelbergh, D. Conformal and Atomic Characterization of Ultrathin CdSe Platelets with a Helical Shape. Nanoletters 2014, 14, 6257-6262.

(32) Jana, S.; De Frutos, M.; Davidson, P.; Abécassis, B. Ligand-Induced Twisting of Nanoplatelets and Their Self-Assembly into Chiral Ribbons. Sci. Adv. 2017, 3: e1701483.

(33) Sayevich, V.; Guhrenz, C.; Dzhagan, V. M.; Sin, M.; Werheid, M.; Cai, B.; Borchardt, L.; Widmer, J.; Zahn, D. R. T.; Brunner, E.; Lesnyak, V.; Gaponik, N.; Eychmüller, A. Hybrid NButylamine-Based Ligands for Switching the Colloidal Solubility and Regimentation of Inorganic-Capped Nanocrystals. ACS Nano 2017, 11, 1559-1571.

(34) Kirkwood, N.; Monchen, J. O. V.; Crisp, R. W.; Grimaldi, G.; Bergstein, H. A. C.; du Fossé, I.; van de Stam, W.; Infante, I.; Houtepen, A. J. Finding and Fixing Traps in II-VI and III-V Colloidal Quantum Dots: The Importance of Z-Type Ligand Passivation. J. Am. Chem. Soc. 2018, 140, 15712-15723.

(35) Izquierdo, E.; Robin, A.; Keuleyan, S.; Lequeux, N.; Lhuillier, E.; Ithurria, S. Strongly Confined HgTe 2D Nanoplatelets as Narrow near Infrared Emitters. J. Am. Chem. Soc. 2016, 138, 10496-10501.

(36) Zhou, Y.; Buhro, W. E. Reversible Exchange of L-Type and Bound-Ion-Pair X-Type Ligation on Cadmium Selenide Quantum Belts. J. Am. Chem. Soc. 2017, 139, 12887-12890.

(37) Yao, Y.; Zhou, Y.; Sanderson, W. M.; Loomis, R. a.; Buhro, W. E. Metal-Halide-Ligated Cadmium Selenide Quantum Belts by Facile Surface Exchange. Chem. Mater. 2018, 30, 28482857.

(38) Naumkin, A. V.; Kraut-Vass, A.; Gaarenstroom, S. W.; Powell, C. J. NIST X-Ray Photoelectron Spectroscopy Database, 2012.

(39) Brammer, L.; Bruton, E. a.; Sherwood, P. Understanding the Behavior of Halogens as Hydrogen Bond Acceptors. Cryst. Growth Des. 2001, 1, 277-290.

(40) Rabouw, F. T.; Van Der Bok, J. C.; Spinicelli, P.; Mahler, B.; Nasilowski, M.; Pedetti, S.; Dubertret, B.; Vanmaekelbergh, D. Temporary Charge Carrier Separation Dominates the Photoluminescence Decay Dynamics of Colloidal CdSe Nanoplatelets. Nano Lett. 2016, 16, 2047-2053.

(41) Bouet, C.; Mahler, B.; Nadal, B.; Abecassis, B.; Tessier, M. D.; Ithurria, S.; Xu, X.; Dubertret, B. Two-Dimensional Growth of CdSe Nanocrystals, from Nanoplatelets to Nanosheets. Chem. Mater. 2013, 25, 639-645.

(42) Armon, S.; Efrati, E.; Kupferman, R.; Sharon, E. Geometry and Mechanics in the Opening of Chiral Seed Pods. Science. 2011, 333, 1726-1730.

(43) Bertolotti, F.; Dirin, D. N.; Ibáñez, M.; Krumeich, F.; Cervellino, A.; Frison, R.; Voznyy, O.; Sargent, E. H.; Kovalenko, M. V.; Guagliardi, A.; Masciocchi, N. Crystal Symmetry Breaking and Vacancies in Colloidal Lead Chalcogenide Quantum Dots. Nat. Mater. 2016, 15, 987-994.

(44) Chen, D.; Gao, Y.; Chen, Y.; Ren, Y.; Peng, X. Structure Identification of Two-Dimensional Colloidal Semiconductor Nanocrystals with Atomic Flat Basal Planes. Nano Lett. 2015, 15, $4477-4482$. 
(45) International Tables for Crystallography Volume C; Prince, E., Ed.; Kluwer academic publishers.

(46) Chu, A.; Livache, C.; Ithurria, S.; Lhuillier, E. Electronic Structure Robustness and Design Rules for 2D Colloidal Heterostructures Electronic Structure Robustness and Design Rules for 2D Colloidal Heterostructures. J. Appl. Phys. 2018, 123, 035701.

(47) Rajadell, F.; Climente, J. I.; Planelles, J. Excitons in Core-Only, Core-Shell and Core-Crown CdSe Nanoplatelets: Interplay between in-Plane Electron-Hole Correlation, Spatial Confinement, and Dielectric Confinement. Phys. Rev. B 2017, 96, 035307.

(48) Zhou, Y.; Wang, F.; Buhro, W. E. Large Exciton Energy Shifts by Reversible Surface Exchange in 2D II-VI Nanocrystals. J. Am. Chem. Soc. 2015, 137, 15198-15208.

(49) Dai, X.; Zhang, Z.; Jin, Y.; Niu, Y.; Cao, H.; Liang, X.; Chen, L.; Wang, J.; Peng, X. SolutionProcessed, High-Performance Light-Emitting Diodes Based on Quantum Dots. Nature 2014, 515, 96-99.

Table of Contents:

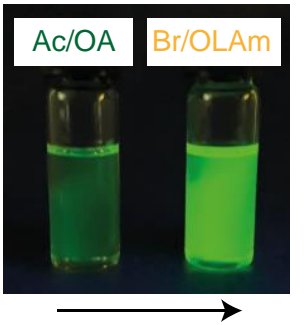

Ligand exchange
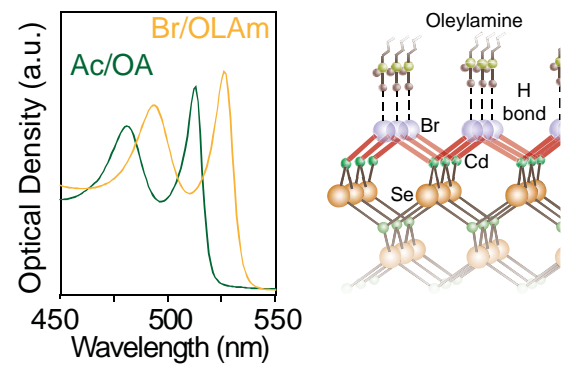